\title{
Benchmarking a Self-Consistent Field Theory for Small Amphiphilic Molecules
}

\author{
Russell B. Thompson* \\ Department of Physics and Astronomy, \\ University of Waterloo, 200 University Avenue West, \\ Waterloo, Ontario, Canada N2L $3 G 1$ and \\ Waterloo Institute for Nanotechnology, \\ University of Waterloo, 200 University Avenue West, \\ Waterloo, Ontario, Canada N2L 3G1 \\ T. Jebb \\ Nanotechnology Engineering Program, \\ University of Waterloo, 200 University Avenue West, \\ Waterloo, Ontario, Canada N2L 3G1 \\ Y. Wen \\ Department of Pure Mathematics, University of Waterloo, \\ 200 University Avenue West, Waterloo, Ontario, Canada N2L 3G1†
}

\begin{abstract}
A minimalist self-consistent field theory for small amphiphilic molecules is presented. The equations for this model are less involved than those for block copolymers and are easily implemented computationally. A new convergence technique based on a variant of Anderson mixing is also presented which allows the equations to be solved more rapidly than block copolymer self-consistent field theory. The computational speed up and simplicity of equations result from a lack of configurational degrees of freedom in the amphiphilic molecular model. The omission of polymeric flexibility leads to qualitatively different predictions compared to known diblock copolymer behaviour.
\end{abstract}

\footnotetext{
*Electronic address: thompson@uwaterloo.ca

$\dagger$ †resent address: Dept of Math \& Stat, McGill Univ, 805 Sherbrooke West, Montreal, QC, Canada H3A 0B9
} 


\section{INTRODUCTION}

The self-assembly of amphiphilic molecules is of fundamental importance in biology and nanotechnology [1]. Amphiphilic block copolymers are being intensely researched, both experimentally and theoretically, for nanotechnological applications [2-4]. While a full array of theoretical tools have been used to understand amphiphilic block copolymer self-assembly, self-consistent field theory (SCFT) has been of particular utility — see for example references 5-9 and references therein. SCFT is well suited to studying long polymer molecules and is computationally more tractable than many simulation approaches [10]. In contrast, amphiphilic surfactants and lipids have not been as extensively studied using SCFT. Surfactants and lipids have in common that they are much smaller than block copolymers, with more complicated and restricted internal degrees of freedom. As such, the computational burden of a faithful SCFT representation can be prohibitive and the fundamental mean field approximation of SCFT becomes less reliable.

Nonetheless, important SCFT contributions have been made in the study of small amphiphilic molecules. Schmid and Schick developed a form of SCFT based on connected hard rods in which density functional theory was used to enforce excluded volume $[11,12]$. This application was confined to a one dimensional analysis of Langmuir monolayers because the method was computationally demanding. Szleifer et al. also confined their SCFT method to one dimensional, spherically symmetric solutions for amphiphilic aggregates [13-16]. Whitmore et al. have developed a lattice-based SCFT for lipid molecules with compressibility [17-19]. This approach is valuable in studies of bilayers. Another popular lattice-based approach is that of Scheutjens, Fleer, Leermakers et al. [20-22]. Off-lattice, Gaussian string SCFT methods for lipids have been developed by Lee, Mezzenga and Fredrickson on the one hand [23, 24], and Schick, Müller, Katsov and Lee on the other [25-32]. These methods use a coarse-graining philosophy wherein the behaviour of lipid self-assembly is understood in terms of a generic amphiphilic molecule, specifically, a block copolymer [33]. Surfactant behaviour has also been examined using this SCFT block copolymer stand-in philosophy [34]. Using such a model for lipids, Katsov, Müller, Schick and Lee (hereafter referred to as KMSL) have made a number of contributions towards understanding behaviours of lipid membranes. In particular, Katsov, Müller and Schick predict a novel pathway for membrane fusion $[25,26]$. Comparisons with simulations using widely different models for lipid 
molecules show a universality of behaviour that is in agreement with their approach [25]. Other dynamic $[6,7,9,35,36]$ or lower dimensional SCFT studies $[5,8,9,37]$ have also pointed towards a universality of behaviour between lipids and amphiphilic block copolymers.

There are issues with the philosophy of KMSL. First, using a Gaussian chain block copolymer as a model for a small amphiphilic molecule grossly overestimates the configurational degrees of freedom. Second, for complex lipid mixtures, such as studied experimentally by Katsaras et al. [38], fully 3D predictions are required. Copolymer-based dynamic 3D SCFT becomes computationally intensive and does not necessarily reveal the ultimately stable phases required for many applications. Even for equilibrium calculations, KMSL have, for the most part, confined themselves to special geometries so as to reduce computational costs. Third, steric and orientational effects that act on length scales too small to be of relevance for many block copolymers are important for smaller amphiphilic molecules. Fourth, the mean field approximation of SCFT works well for large polymeric molecules but will be worse for small amphiphilic molecules.

In this paper, we address the first two of these points. The KMSL approach has been validated in some contexts by other methods [25,33]. This lends credibility to the idea of keeping the excessive Gaussian chain configurational degrees of freedom as a simple and pragmatic way to enable an SCFT calculation. We present as an alternative the other extreme, where all the polymeric degrees of freedom are removed. This has two advantages. First, by removing configurational freedom, we greatly reduce the computational burden, allowing us to conduct larger scale and less restricted computations. In polymer SCFT, the main computational burden is the repeated solution of modified diffusion equations. These equations vanish in our model and are replaced by simple convolution integrals. Second, since our model will allow us to adjust internal degrees of freedom continuously, we can qualitatively reproduce block copolymer behaviour on the one hand, lending further evidence in support of KMSL in some circumstances, and on the other we can show that fundamentally different physics can dictate behaviour when internal molecular degrees of freedom are suppressed.

These results will be demonstrated through the use of a deliberately artificial environment. Block copolymer physics is based fundamentally on packing frustration [39]. Packing frustration is most clearly manifested in pure copolymer melts, so a logical test arena for dis- 
tinguishing possible qualitative differences of behaviour between molecules with and without configurational entropy would be a pure melt system. Such an environment is not realistic for small amphiphilic molecules. Computationally, however, this poses no problem. By examining a deliberately artificial system of pure small amphiphilic molecules, we subject our model to the most severe test. If, under these conditions, we find behaviour qualitatively the same as for block copolymers, then we can be fairly certain that for milder and more realistic situations, a block copolymer model of small amphiphilic molecules should be good for most coarse, qualitative predictions. On the other hand, if the fundamental physics is found to be different, then one must be on the lookout for situations where qualitative predictions of a block copolymer stand-in model might fail.

We find that both situations can occur. In our simple model, allowing some internal vibrational degree of freedom, we get qualitatively the same behaviour as for diblock copolymers. For small amphiphilic molecules that might map onto this situation, the KMSL approach is appropriate. Nonetheless, even for this case our method still has computational advantages. If the stiffness of the internal vibrational motion is turned up very high so as to be suppressed, the results are qualitatively inconsistent with block copolymers. This does not invalidate the KMSL model, but it does provide evidence that there could be exotic situations where the fundamental physics governing the qualitative behaviour of small amphiphilic molecules might not be the same as for block copolymers. Such situations could become increasingly important in circumstances with, for example, nanoparticles or biological inclusions in membranes, where excessive configurational degrees of freedom could change results dramatically. An example of such an exotic situation has already been reported by us in the context of polymer systems [40].

\section{THEORY}

Small amphiphilic molecules are modelled as two repulsive point particles, each with a chosen volume and potential, bound together by a connectivity potential which is characterized by a spring constant $k$ - see the schematic in figure 1 . The parameter $k$ gives the vibrational stiffness of the molecule. For small values of $k$, the "head" and "tail" groups can move somewhat with respect to each other, coarsely representing changing conformations of the amphiphilic molecule. For large values of $k$, the relative head and tail positions are fixed 


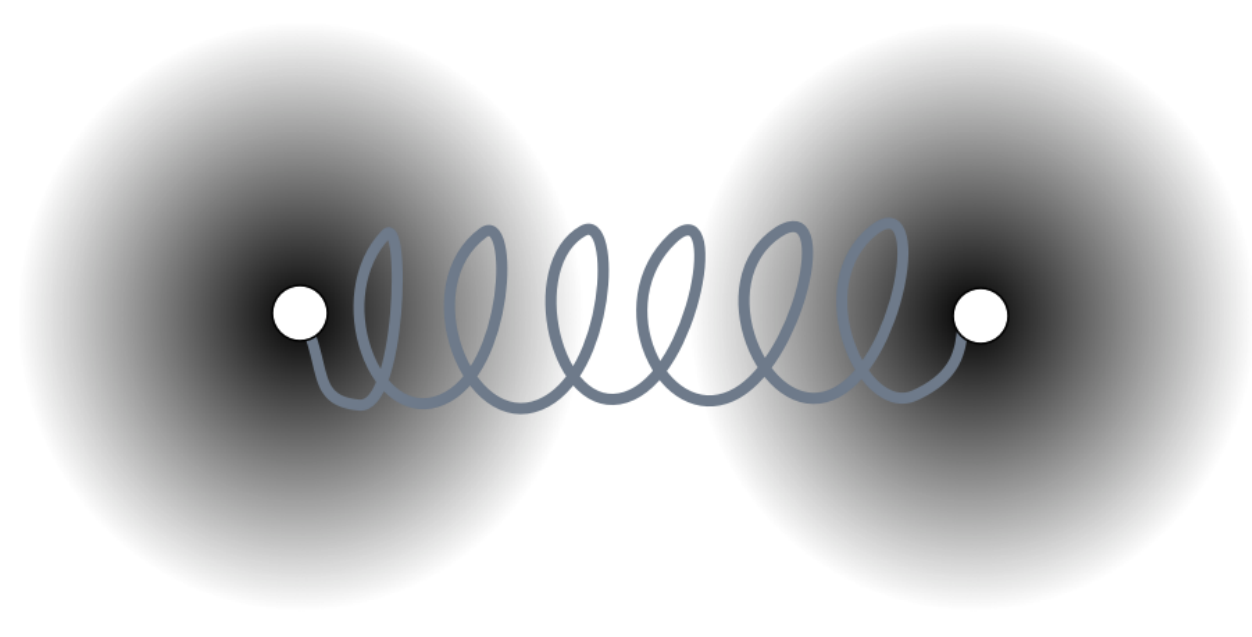

FIG. 1: Schematic of small amphiphilic molecular model. The shaded zones correspond to the potentials of the interaction sites, which are themselves shown as white dots. Each of the two sites is assigned, in general, a different volume and potential in order to give it coarse features corresponding to various lipid or surfactant molecules.

within the molecule, representing an amphiphilic molecule with little or no internal degrees of freedom. The SCFT equations are derived through a standard path integral formalism given in the appendix. The resulting four equations are

$$
\begin{aligned}
w_{H}(\mathbf{r}) & =\int d \mathbf{r}^{\prime} \mathcal{V}_{H T}\left(\left|\mathbf{r}-\mathbf{r}^{\prime}\right|\right) \varphi_{T}\left(\mathbf{r}^{\prime}\right)-\tilde{\kappa}\left[1-\varphi_{H}(\mathbf{r})-\varphi_{T}(\mathbf{r})\right] \\
w_{T}(\mathbf{r}) & =\int d \mathbf{r}^{\prime} \mathcal{V}_{H T}\left(\left|\mathbf{r}-\mathbf{r}^{\prime}\right|\right) \varphi_{H}\left(\mathbf{r}^{\prime}\right)-\tilde{\kappa}\left[1-\varphi_{H}(\mathbf{r})-\varphi_{T}(\mathbf{r})\right] \\
\varphi_{H}(\mathbf{r}) & =\frac{\phi_{H} V}{Q} \int d \mathbf{r}^{\prime} G\left(\mathbf{r}, \mathbf{r}^{\prime}\right) \\
\varphi_{T}(\mathbf{r}) & =\frac{\phi_{T} V}{Q} \int d \mathbf{r}^{\prime} G\left(\mathbf{r}^{\prime}, \mathbf{r}\right)
\end{aligned}
$$

where $G\left(\mathbf{r}, \mathbf{r}^{\prime}\right)$ is

$$
G\left(\mathbf{r}, \mathbf{r}^{\prime}\right)=\exp \left[-\phi_{H} w_{H}(\mathbf{r})-\phi_{T} w_{T}\left(\mathbf{r}^{\prime}\right)-\mathcal{V}_{\text {conn }}\left(\left|\mathbf{r}-\mathbf{r}^{\prime}\right|\right)\right]
$$

and $Q$ is

$$
Q=\int d \mathbf{r} d \mathbf{r}^{\prime} G\left(\mathbf{r}, \mathbf{r}^{\prime}\right)
$$

$H$ denotes head groups, $T$ tail groups and $V$ is the calculation volume in the canonical ensemble. In equations (1) and (2), $\mathcal{V}_{H T}(r)$ is the (effective) repulsive interaction between 
heads and tails of distinct molecules. $\mathcal{V}_{\text {conn }}(r)$ in equation $(5)$ for the propagator $G\left(\mathbf{r}, \mathbf{r}^{\prime}\right)$ is the connectivity potential between heads and tails within a single molecule. These two potentials will in general be chosen to reflect properties of the amphiphilic molecules. As described in the appendix, we have chosen Gaussian forms for them. The prefactor and width of the $\mathcal{V}_{H T}(r)$ Gaussian are given by $\mathcal{V}_{H T}^{0}$ and $\mathcal{V}_{H T}^{w}$, respectively. While the nonlocal nature of the potential $\mathcal{V}_{H T}(r)$ is distinct from the typical implementation of modern SCFT, non-local potentials have also been extensively used in SCFT in the form of square gradient terms. The seminal works of Hong, Whitmore and Noolandi are good examples of this $[41,42]$. In our case, the non-local potential is essential for physical results since the molecules have negligible non-locality in their architecture, unlike extended polymer molecules. In the polymer case, it is this non-local architecture that gives the required non-locality to the problem so that interfaces are not trivial step functions. For us, the potential provides this important non-locality. The volume fractions of heads and tails as a function of position are given by $\varphi_{H}(\mathbf{r})$ and $\varphi_{T}(\mathbf{r})$, respectively, whereas the global volume fractions of heads and tails are given by $\phi_{H}$ and $\phi_{T}$. $Q$ is the partition function of a single amphiphilic molecule subject to the fields $w_{H}(\mathbf{r})$ and $w_{T}(\mathbf{r})$, and these are in turn the chemical potential fields of the heads and tails, respectively. Finally, $\tilde{\kappa}$ is a parameter that enforces a constant overall density in the liquid, that is, incompressibility. These equations are solved self-consistently, as described in the Computational section, to provide $\varphi_{H}(\mathbf{r})$ and $\varphi_{T}(\mathbf{r})$ as output; that is, the equilibrium or meta-stable morphologies of the amphiphilic system. The free energy of a given morphology found in this way is

$$
\begin{aligned}
\frac{F}{n k_{B} T}= & -\ln Q+\frac{1}{V} \int d \mathbf{r}\left\{\int d \mathbf{r}^{\prime} \varphi_{H}(\mathbf{r}) \mathcal{V}_{H T}\left(\left|\mathbf{r}-\mathbf{r}^{\prime}\right|\right) \varphi_{T}\left(\mathbf{r}^{\prime}\right)\right. \\
& \left.+\frac{\tilde{\kappa}}{2}\left[1-\varphi_{H}(\mathbf{r})-\varphi_{T}(\mathbf{r})\right]^{2}-w_{H}(\mathbf{r}) \varphi_{H}(\mathbf{r})-w_{T}(\mathbf{r}) \varphi_{T}(\mathbf{r})\right\} \\
& +\ln \left[V \int d \mathbf{r} e^{-\mathcal{V}_{\text {conn }}(\mathbf{r})}\right]
\end{aligned}
$$

with a uniform limit of

$$
\frac{F}{n k_{B} T}=\phi_{H} \phi_{T} \int d \mathbf{r} \mathcal{V}_{H T}(\mathbf{r})
$$

All volumes in equations (1)-(8) are made dimensionless with the molecular volume $v_{0}$ as explained in the appendix.

Input parameters are $\phi_{T}, k, l, \mathcal{V}_{H T}^{0}$ (prefactor of the Gaussian form), $\mathcal{V}_{H T}^{w}$ (width of the Gaussian form), $\tilde{\kappa}$ and $V$. The volume $V$ is not a physical parameter, and $\tilde{\kappa}$ is not arbitrary 
since it should be taken as large as possible to enforce incompressibility. For this work, we fix the spring equilibrium length at $l=1 \sigma$ and the head-tail Gaussian potential width at $\mathcal{V}_{H T}^{w}=0.5 \sigma$. As mentioned in the appendix, we choose the volume of one molecule $v_{0}$ as a reference volume, $\sigma \equiv v_{0}^{1 / 3}$ as our length scale and we make all potentials dimensionless with the thermal energy $k_{B} T$ where $k_{B}$ is Boltzmann's constant and $T$ is the temperature. This leaves just three parameters: $\phi_{T}, k$ and $\mathcal{V}_{H T}^{0}$. Because of incompressibility, $\phi_{H}$ is not independent. Of course, one could choose to adjust the other parameters which are available to more accurately describe shapes and interactions of different amphiphilic molecules. One could also choose different, more realistic, potentials. Since lipid and surfactant molecules are, in general, so much smaller than block copolymers, it makes sense that there are more parameters necessary to specify the present model. Block copolymer molecular features and potentials are negligible on the length scale of the polymeric molecule. That is not the case

for smaller amphiphilic molecules. Nonetheless, the model has in common with KMSL a minimalist amphiphilic philosophy, so certain molecular features are not represented. For example, any rod-like rigidity in the molecule will be lost. Due to the incompressibility method of enforcing excluded volume, the particularly shape of the molecular constituents is not fixed. Thus the molecules are truly soft and deformable depending on the morphology that they form, and will not act completely as point masses as implied in figure (1).

\section{COMPUTATIONAL}

We surveyed a two dimensional parameter space, varying $\phi_{T}$ from 0 to 1 and $\mathcal{V}_{H T}^{0}$ from 10 to 50 , for two values of vibrational stiffness, $k=10$ and $k=1000$. For each point of this parameter space, one would ideally optimize the size and shape of the calculational box so as to remove commensurability effects due to the periodic nature of the amphiphilic morphologies. We decided however to use a larger box to relieve commensurability effects since this is more scalable for possible later work involving amphiphilic molecules in solution. For such cases, predicted morphologies may not be strongly periodic, and a large box size would be a more appropriate approach. For the present system, the large box is supplemented with two other, smaller, box sizes in order to clarify cases where many defects might be present in the larger box. Although this method does not allow for the construction of rigorous phase diagrams, it does allow for the qualitative phase behaviour of the system to be understood. 
Since this system is deliberately fictional in that amphiphilic molecules do not self-assemble in a pure, solvent free, environment, the exact details of such phase maps are not required in any case. We chose cubical boxes with sides of length $5 \sigma, 7.5 \sigma$ and $10 \sigma$, respectively. For each of these three box sizes, we did three independent runs starting from different random configurations. About 2000 runs were done in total for both two dimensional $\phi_{T} \times \mathcal{V}_{H T}^{0}$ parameter spaces corresponding to $k=10$ and $k=1000$. This gave us a good, qualitative, picture of the behaviours of these systems.

The algorithm we used for solving equations (1)-(6) is as follows. We discretized the equations on a three dimensional, regularly spaced grid. For this work, we found that a discretization of $64^{3}$ was a good compromise between accuracy and speed. We made a guess for the volume fractions $\varphi_{H / T}(\mathbf{r})$ and substituted these into equations (1) and (2). This required the Fourier transform $\bar{\varphi}_{H / T}(\mathbf{k})$. The fields $w_{H / T}(\mathbf{r})$ were then exponentiated (equation (5)), each of these was Fourier transformed and equation (6) was solved for $Q$ through a convolution integral and then integrating over space using simple sums. For periodic boundary conditions, simple sums have exponential order and no improvement can be had from more complicated Newton-Cotes quadratures [43]. Equations (3) and (4) were solved by using $Q$ and the same convolution integral results. There were therefore two pairs of Fourier transforms in each iteration of the algorithm. The resulting $\varphi_{H / T}(\mathbf{r})$ volume fractions were mixed in a superposition with the original ones using Picard iteration until convergence was complete. We used a self-consistency criterion of less than one part in $10^{6}$ difference between input and output $\varphi_{H / T}(\mathbf{r})$ volume fractions according to the square of a relative $L^{2}$ norm.

The reader might notice that we used a different incompressibility condition than is generally employed in block copolymer SCFT. Normally, a pressure field $\xi(\mathbf{r})$ is included as a Lagrange multiplier to enforce incompressibility. The above algorithm using the $\xi(\mathbf{r})$ pressure field is unstable except for very coarse grids. The algorithm is stable however when using a quadratic energy penalty proportional to $\tilde{\kappa}$ to enforce incompressibility. This method was originated by Helfand [44] and continues to be used due to its favourable numerical properties $[6,36,45]$. We found that a value of $\tilde{\kappa}=1000$ created an energy penalty sufficient to keep a total volume fraction of 1 to within approximately half a percent. Using this approach, the equations can be iterated on either the fields $w_{H / T}(\mathbf{r})$ or the volume fractions $\varphi_{H / T}(\mathbf{r})$. We found a slight benefit to iterating on the volume fractions and judge this approach to 
be more scalable in terms of possible future work involving dynamics.

Although the energy penalty approach is stable, the equations are very stiff, meaning we had to use a small mixing parameter for the Picard iteration resulting in many iterations for convergence. Fortunately, the SCFT equations are continuous with respect to the energy penalty parameter $\tilde{\kappa}$, so the equations can be solved in stages. We began with $\tilde{\kappa}=10$, which allowed a large mixing parameter of 0.1 to be used for an initial solution of the SCFT equations from a random input. Then, this output, which did not satisfy incompressibility, was used for another stage of the computation with $\tilde{\kappa}=100$ and a mixing parameter of 0.01. Finally, this was in turn used as the input for a final stage with $\tilde{\kappa}=1000$ and a mixing parameter of 0.001 . This process resulted in significantly faster convergence and ultimately satisfied incompressibility.

Within each $\tilde{\kappa}$ stage of the convergence, there were iterations where the convergence levelled off and we were unable to compute a satisfactory self-consistent solution. To resolve this, we here introduce a new form of Anderson iteration. Anderson iteration is a linear extrapolation technique, based on a saved history of previous iterations, that can speed block copolymer convergence enormously [43, 46]. For other non-block copolymer SCFT however, it can become unstable and fail. One of us previously introduced a method in which single Anderson steps were interleaved with clumps of Picard iteration steps in order to "punch though" stalled patches of convergence $[47,48]$. This is in contrast to a previous block copolymer implementation that switched from exclusive Picard iteration to exclusive Anderson iteration [46]. These occasional, single, Anderson steps were taken regularly and accepted only if they improved the convergence status; they were rejected otherwise [47, 48]. This method unfortunately doesn't work for the present set of amphiphilic SCFT equations, but it does form the basis of our new approach. In this approach, a single Anderson step was taken if the convergence rate dropped below some threshold. We used a different threshold for each $\tilde{\kappa}$ stage of the convergence: the square of a relative $L^{2}$ norm change in the iterated value of $\varphi_{H / T}(\mathbf{r})$ of less than $1 \%$ for $\tilde{\kappa}=10$ or 100 prompted an Anderson step. For $\tilde{\kappa}=1000$, we used a threshold of $0.1 \%$. Unlike references 47 and 48, we kept the Anderson step even if it worsened the level of convergence. Indeed, that was the usual outcome. The large Anderson step normally exceeded the stability limit, but the convergence would subsequently "heal" after some Picard iterations to a level better than that at which it was previously stuck. In some cases, the convergence improvement after a single Anderson step was up to an order 


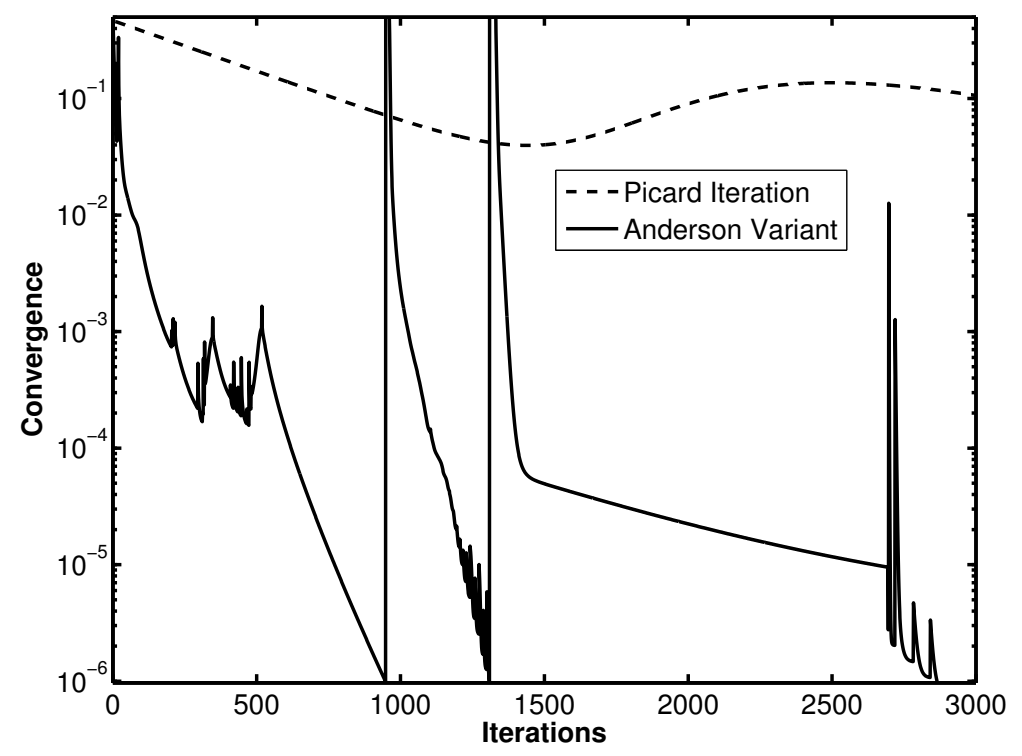

FIG. 2: Convergence vs iterations for Picard iteration (dashed line) and new, stepped Anderson variant (solid line).

of magnitude. More typically however, the "unstable" Anderson step provided a means to punch through a convergence sticking point. The use of a combination of a large, unstable, explicit step followed by a number of smaller, stable, explicit steps in the convergence of PDEs was demonstrated by Eriksson et al. [49]. We used this idea coupled with the Anderson method of determining a near optimal large step [50]. Taken together, adjusting $\tilde{\kappa}$ in stages and using occasional, single, unstable Anderson steps allowed us to converge the SCFT equations (1)-(6) very rapidly. Without these techniques, the equations would be intractable using an ordinary explicit Picard algorithm. Figure 2 shows the iteration improvement for this approach over regular Picard iteration for a gyroid morphology. In figure 2, one can see two large jumps in the solid curve from a convergence level of $10^{-6}$ (our chosen level of self-consistency) up to $\sim 10^{0}$. This is where we used the output of weaker incompressibility values of $\tilde{\kappa}$ as the input for runs with stronger incompressibility. The smaller and more frequent jumps and noise in the convergence of the solid curve are caused by single Anderson iterations whereas the smooth portions of the curve are the Picard "healing" iterations. The dashed curve shows the same computation starting with a strong incompressibility parameter $\tilde{\kappa}=1000$ and using only Picard iteration. Even within a single 
stage of the solid curve, one can see a clear improvement of convergence trajectory due to the Anderson iterations. For the results shown in figure 2, and throughout this work, just two iterations were saved in the Anderson history. This was found to give good convergence most of the time, although occasionally, for difficult runs, we used more iterations in the history [65]. More information on the particulars of how Anderson iteration works and is implemented can be found in references $[43,46,50]$. It should be noted that Anderson mixing is quite straightforward to implement and is completely modular. That is, the subroutine is unaffected by the nature of the equations to be solved and so can be easily applied to a variety of problems throughout science and engineering.

The fields and volume fractions of the converged SCFT equations (1)-(6) were used in equation (7) to find the free energies of competing structures. We estimate our free energies to be accurate to half a percent for $k=10$ and about three percent for $k=1000$. The limiting factor for accuracy was the spatial grid size. This level of accuracy in the free energies was not enough to determine subtle features in phase maps, but was sufficient to give a qualitative sense of system behaviours. One could of course choose to increase the accuracy, but as mentioned, this is not helpful for the present fictional system. Lastly, since all runs can be done independently, the parameter space surveys were easily parallelized through a straightforward serial farming approach.

\section{RESULTS AND DISCUSSION}

For a "loose" spring constant of $k=10$, we found phases consistent with diblock copolymers and in the same qualitative order. Specifically, as we varied $\phi_{T}$ from 0 to 1 and $\mathcal{V}_{H T}^{0}$ from 10 to 50, we found body-centered cubic spheres (S), hexagonally packed cylinders (C), bicontinuous gyroid $(\mathrm{G})$, perforated lamellar $(\mathrm{PL})$ and lamellar phases $(\mathrm{L})$ in qualitatively the same locations of volume fraction-segregation parameter space as for diblock copolymers [39]. Examples of our results at $k=10$ and $\mathcal{V}_{H T}^{0}=30$ are shown in figure 3. In diblock copolymers, the $\mathrm{G}$ phase is stable rather than PL but they are very close in free energy, so it is not surprising to observe both here [66]. This is indicative that the present model can be made to behave in qualitatively the same way as diblock copolymers and thus can be used as a minimalist model of amphiphilic molecules in the same spirit as KMSL. The computational reduction realized through not having to repeatedly solve modified diffusion 


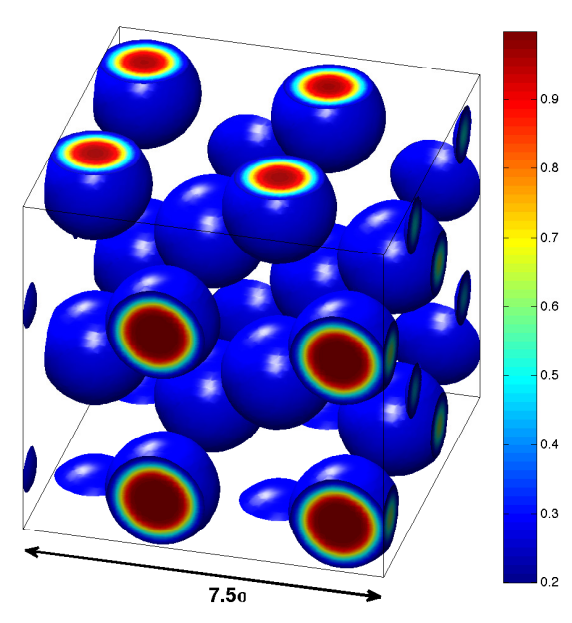

(a)

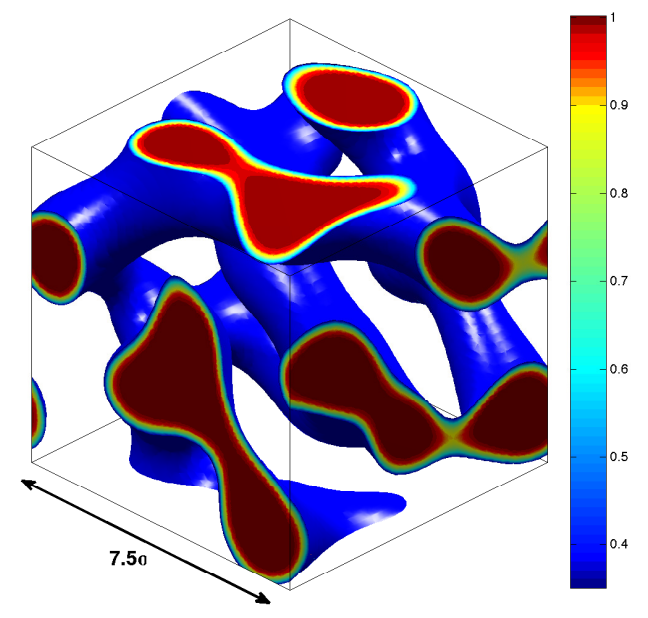

(c)

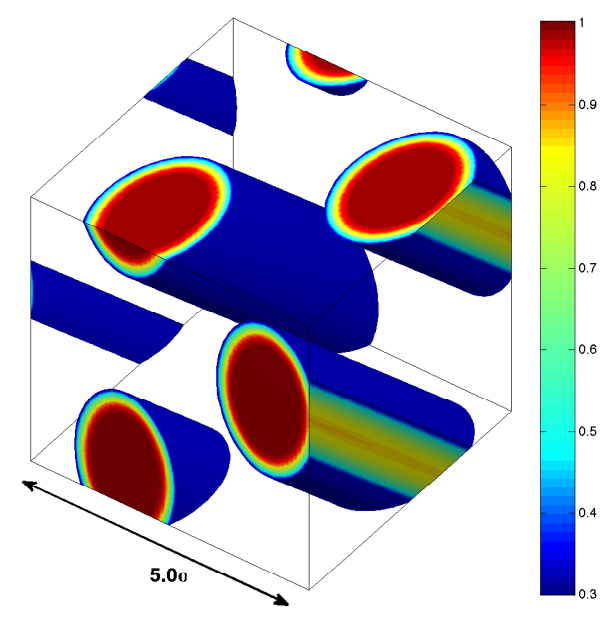

(b)

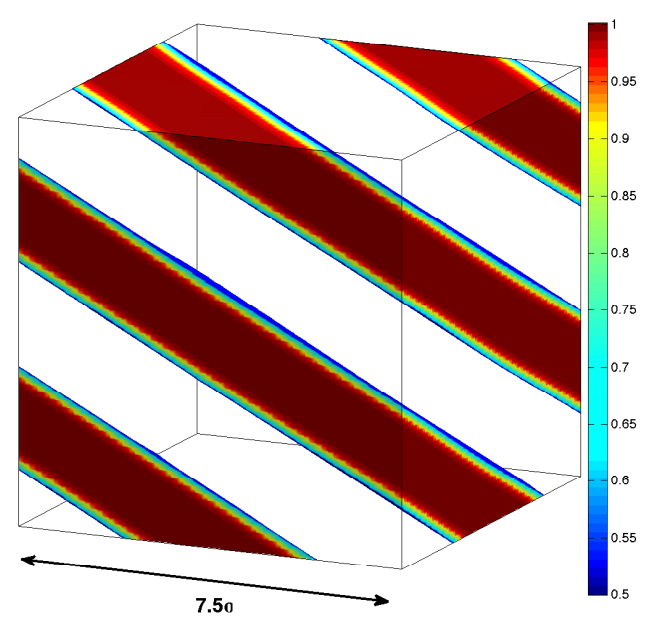

$(d)$

FIG. 3: Tail group densities of sample morphologies at $k=10$ and $\mathcal{V}_{H T}^{0}=30$. (a) $\phi_{T}=0.2$ (body centre cubic spheres) (b) $\phi_{T}=0.3$ (hexagonally packed cylinders) (c) $\phi_{T}=0.35$ (bicontinuous, three-point coordinated complex phase) (d) $\phi_{T}=0.5$ (lamellae). For each panel, the isosurfaces are drawn at the value of $\phi_{T}$. Contour plots on faces show internal density profiles according to the colorbars.

equations means that this model could be a complementary approach to KMSL allowing comparatively large cells in full three dimensions to be examined. Also, the SCFT equations for this model are much simpler to implement, allowing for rapid development and 


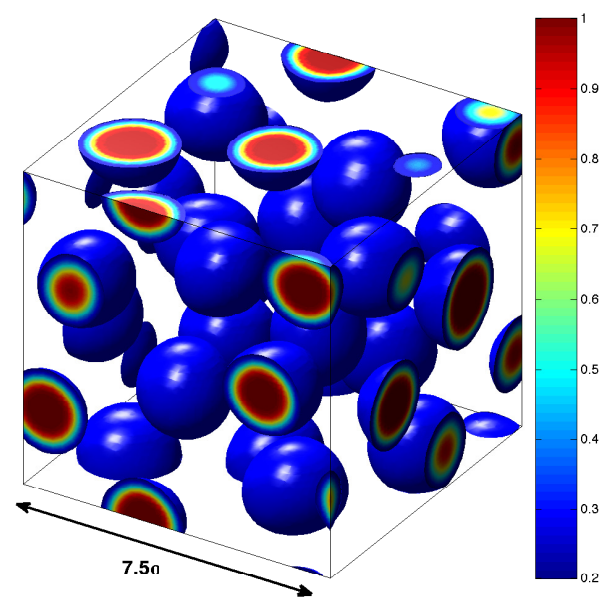

(a)

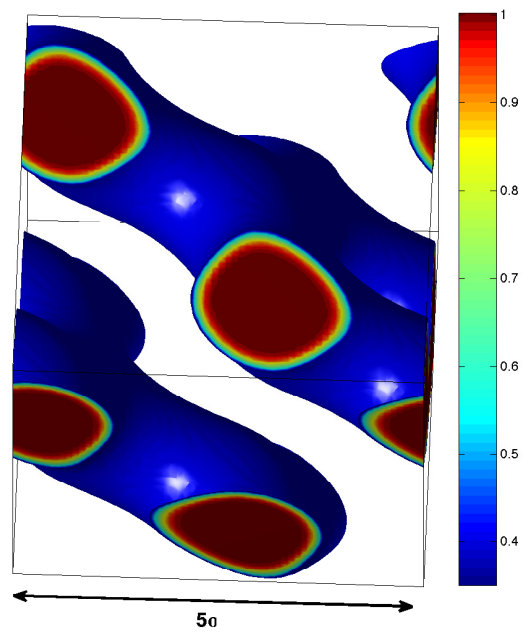

(c)

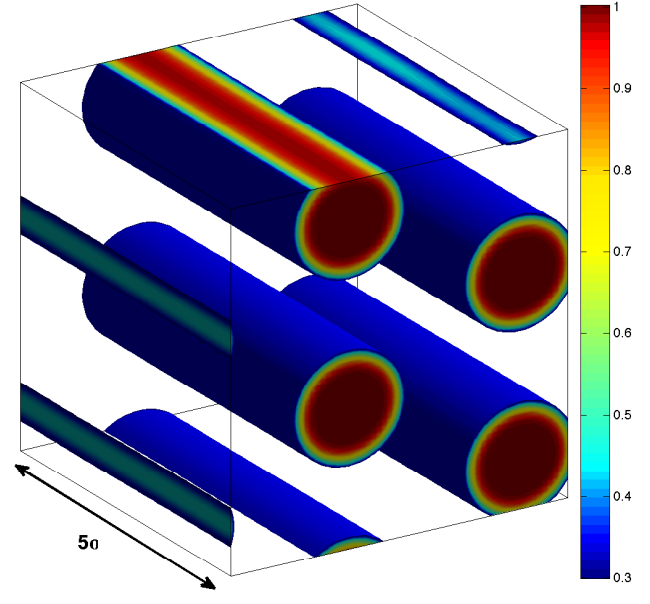

(b)

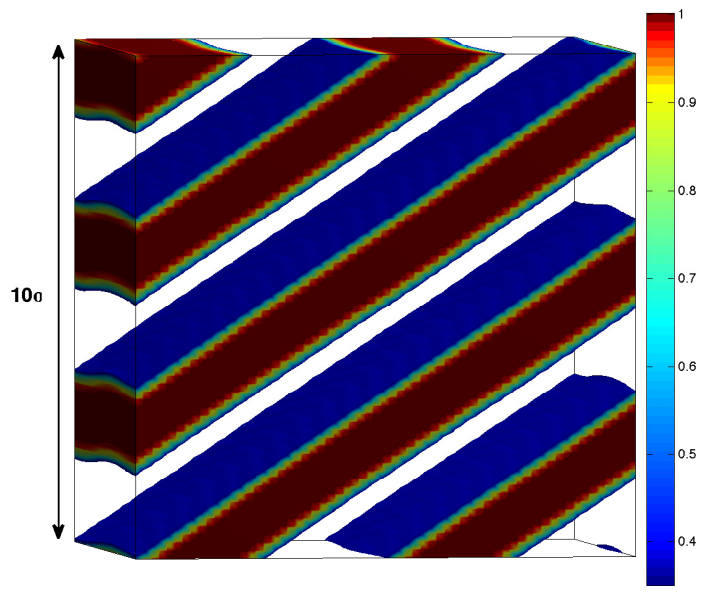

$(d)$

FIG. 4: Tail group densities of sample morphologies at $k=1000$ and $\mathcal{V}_{H T}^{0}=30$, except for panel (c) which is at $\mathcal{V}_{H T}^{0}=40$. (a) $\phi_{T}=0.2$ (disordered spheres) (b) $\phi_{T}=0.3$ (hexagonally packed cylinders) (c) $\phi_{T}=0.35$ (perforated lamellae at $\left.\mathcal{V}_{H T}^{0}=40\right)(\mathrm{d}) \phi_{T}=0.5$ (lamellae). For each panel, the isosurfaces are drawn at the value of $\phi_{T}$. Contour plots on faces show internal density profiles according to the colorbars.

modification of code.

For a stiff spring constant of $k=1000$, the classical S, C and L phases were still found. Examples of our results at $k=1000$ and $\mathcal{V}_{H T}^{0}=30$ are shown in figure 4 . The presence of 
the complex PL and G phases was greatly reduced, with the PL phase appearing only at higher segregations and for narrow regions of the phase diagram. The G phase was observed only rarely and always with a detectably higher free energy than the PL phase. In addition, many results showed highly defected structures with almost identical free energies to the stable phases. Thus the free energy landscape seems to have been splintered compared to the $k=10$ or block copolymer situations, with many defected morphologies with similar free energies competing with each other. This was seen most clearly in the S phase which ceased to be always BCC for $k=1000$. A disordered spherical phase was found which we assume is a defected close packed morphology. A comparison between $k=10$ and $k=1000$ S morphologies, with all other parameters equal, showed that the spheres in the two cases had the same maximum internal volume fractions and edge profiles. The only differences were that the spheres in the $k=1000$ case had smaller diameters and were more densely packed. Specifically, for $\mathcal{V}_{H T}^{0}=30$ and $\phi_{T}=0.2$, we counted 4 spheres in a cubic box of side $5 \sigma$ for $k=10$ compared to 8 spheres in the same size box for $k=1000$.

These changes in self-assembly behaviour are consistent with the removal of the main physics of block copolymers, namely, packing frustration. Packing frustration for block copolymers is the tendency to form morphologies with constant thickness interfaces so as to reduce polymer stretching [39]. For example, the stability domain of the G phase at high segregations is expected to be reduced because the strong stretching of polymer molecules causes polymeric configurational entropy to be of less importance [51]. Similarly, the S phase adopts a BCC configuration in diblock copolymers since this stretches the polymers less than a closed packed configuration. The results for our model with $k=1000$ provides evidence that structures tend to be determined more locally than globally. That is, they prefer to have constant mean curvature in order to reduce interfacial energy; this is the dominant physical mechanism for small amphiphilic molecules with minimal configurational degrees of freedom. There is still packing frustration of a sort, namely that space must be filled, but this does not have the same implications as block copolymer packing frustration [52].

The idea of turning the connectivity stiffness up even higher may tempt the idea of using this model to represent amphiphilic hard rods, however this must be approached with caution. Although some generic amphiphilic behaviour common with the hard rod system may be reproduced, the particularly interesting liquid crystal phases are beyond such a model. This is due to the use of an ensemble averaged excluded volume. More complicated 
SCFT models, using varieties of classical density functional theory or related techniques, have been successfully used to model rod-coil and semi-flexible polymer systems. Examples of these approaches can be found in references 53-63. These approaches must deal with orientational degrees of freedom, making them much more computationally intensive. They go beyond the spirit of the generic amphiphilic behaviour modelled in KMSL or our present approach.

\section{CONCLUSIONS}

We have introduced a simple SCFT model of small amphiphilic molecules that is computationally much simpler to implement and faster to run than block copolymer SCFT. It makes relatively large volume calculations tractable and thus complements polymeric SCFT and computer simulation approaches to modelling lipid and surfactant molecules. When taken together, the block copolymer and small molecule SCFTs cover two disparate approximations of lipid or surfactant molecules. Specifically, one heavily overestimates the configurational degrees of freedom while the other can be adjusted to ignore them completely. Our model is flexible in that it can qualitatively reproduce the behaviour of diblock copolymers for a suitably weak strength of connectivity potential. For a stronger connectivity potential, the predictions are qualitatively different from diblock copolymer systems due to the suppression of packing frustration. Disordered spheres with a packing higher than that of BCC spheres together with an unstable gyroid phase indicate that for a pure amphiphilic theoretical test system with a strong connectivity potential, the fundamental physics governing the self-assembly is distinct from block copolymers.

Although the SCFT equations for this model are simple, converging them proved, at first, to be non-trivial. The Anderson iteration variant that we introduce here is a simple and modular solution to the stiffness of the SCFT equations. When coupled with a variable incompressibility approach, the equations become rapidly soluble.

In future work, it will be necessary to add a solvent so that more realistic amphiphilic systems can be studied. Multiple solvents could be added to model oil-water-surfactant system equilibriums in large 3D cells. Multiple amphiphilic molecules could be added to study complex vesicle and micelle formation. For very complicated mixtures, it may be possible to take advantage of GPU computing to deal with the FFTs. In particular, it must be 
emphasized that the present results which show a difference in physical mechanism between non-flexible amphiphilic molecules and block copolymers is a deliberately artificial case. The addition of solvent would, in most cases, reduce the severity of the environment with respect to the role of polymer configurational entropy. The present results do not therefore negate the previous findings of KMSL with respect to pore formation and membrane fusion, but rather offer a computationally less demanding method. It is also a method that may be of utility for situations where the inclusion of excessive polymeric degrees of freedom in modelling small amphiphilic molecules may not be appropriate.

\section{Appendix: Derivation of the SCFT Equations}

Consider a system of $n$ amphiphilic molecules. We write a coarse-grained model for these molecules based on the potential

$$
\mathcal{V}_{\text {tot }}=\sum_{i=1}^{n} \mathcal{V}_{\text {conn }}\left(\mathbf{r}_{i}^{H}, \mathbf{r}_{i}^{T}\right)+\sum_{i, j=1}^{n} \mathcal{V}_{H T}\left(\left|\mathbf{r}_{i}^{H}-\mathbf{r}_{j}^{T}\right|\right)+\mathcal{V}_{E O S}\left(\left\{\mathbf{r}^{H}, \mathbf{r}^{T}\right\}\right)
$$

where $H$ denotes head groups and $T$ tail groups. All energies are made dimensionless in this

work by dividing by $k_{B} T$, where $k_{B}$ is Boltzmann's constant and $T$ is temperature. $\mathbf{r}_{i}^{H(T)}$ is the position of a single head (tail) group of molecule $i$. In the third term of equation (A.1), the notation $\left\{\mathbf{r}^{H}, \mathbf{r}^{T}\right\}$ refers to the set of all molecule head and tail group positions. The three terms of (A.1) are, respectively, connectivity of heads and tails, two-body interactions between heads and tails and an equation of state term to enforce incompressibility. $\mathcal{V}_{H H}$ and $\mathcal{V}_{T T}$ terms are possible too, but we can set them to zero since, through incompressibility, they can be removed. The two-body term will be converted later to a field based description using the instantaneous volume fraction operators

$$
\hat{\varphi}_{H(T)}=v_{H(T)} \sum_{i=1}^{n} \delta\left(\mathbf{r}-\mathbf{r}_{i}^{H(T)}\right)
$$

where $v_{H}$ or $v_{T}$ are the volumes of single head or tail groups. The EOS term will be defined only in a field based way. The connectivity term will be left in a particle based description. Therefore we have

$$
\begin{aligned}
\mathcal{V}_{t o t} & =\sum_{i=1}^{n} \mathcal{V}_{\text {conn }}\left(\mathbf{r}_{i}^{H}, \mathbf{r}_{i}^{T}\right)+\mathcal{V}_{H T}\left[\hat{\varphi}_{H}, \hat{\varphi}_{T}\right]+\mathcal{V}_{E O S}\left[\hat{\varphi}_{H}, \hat{\varphi}_{T}\right] \\
& \equiv \sum_{i=1}^{n} \mathcal{V}_{\text {conn }}\left(\mathbf{r}_{i}^{H}, \mathbf{r}_{i}^{T}\right)+\mathcal{V}\left[\hat{\varphi}_{H}, \hat{\varphi}_{T}\right]
\end{aligned}
$$


where, for simplicity, we have defined $\mathcal{V}$ as the sum of the last two terms. The partition function for this system in the canonical ensemble is

$$
\mathcal{Z}=\frac{\mathcal{N}}{n !} \int \prod_{i=1}^{n} d \mathbf{r}_{i}^{H} d \mathbf{r}_{i}^{T} \exp \left[-\sum_{i=1}^{n} \mathcal{V}_{\text {conn }}\left(\mathbf{r}_{i}^{H}, \mathbf{r}_{i}^{T}\right)\right] \exp \left\{-\mathcal{V}\left[\hat{\varphi}_{H}, \hat{\varphi}_{T}\right]\right\}
$$

where $\mathcal{N}$ is a normalization constant arising from kinetic energy terms; it has units of $1 /$ volume $^{2 n}$. Using the delta functional identity

$$
\int \mathcal{D} \Phi \delta[\Phi-\hat{\phi}] F[\Phi]=F[\hat{\phi}]
$$

where $F$ is any functional, equation (A.4) becomes

$$
\begin{aligned}
\mathcal{Z}= & \frac{\mathcal{N}}{n !} \int \mathcal{D} \Phi_{H} \mathcal{D} \Phi_{T} \delta\left[\Phi_{H}-\hat{\phi_{H}}\right] \delta\left[\Phi_{T}-\hat{\phi_{T}}\right] \prod_{i=1}^{n} d \mathbf{r}_{i}^{H} d \mathbf{r}_{i}^{T} \\
& \times \exp \left[-\sum_{i=1}^{n} \mathcal{V}_{\text {conn }}\left(\mathbf{r}_{i}^{H}, \mathbf{r}_{i}^{T}\right)\right] \exp \left\{-\mathcal{V}\left[\Phi_{H}, \Phi_{T}\right]\right\}
\end{aligned}
$$

Now using the delta functional definition

$$
\delta[\Phi-\hat{\phi}]=\int_{-i \infty}^{i \infty} \mathcal{D} W \exp \left[\frac{1}{v_{0}} \int d \mathbf{r} W(\Phi-\hat{\phi})\right]
$$

where $v_{0}$ is a reference volume, the partition function (A.6) becomes

$$
\begin{aligned}
\mathcal{Z}= & \frac{\mathcal{N}}{n !} \int \mathcal{D} \Phi_{H} \mathcal{D} \Phi_{T} \mathcal{D} W_{H} \mathcal{D} W_{T} \prod_{i=1}^{n} d \mathbf{r}_{i}^{H} d \mathbf{r}_{i}^{T} \exp \left[-\sum_{i=1}^{n} \mathcal{V}_{\text {conn }}\left(\mathbf{r}_{i}^{H}, \mathbf{r}_{i}^{T}\right)\right] \\
& \times \exp \left\{-\mathcal{V}\left[\Phi_{H}, \Phi_{T}\right]+\frac{1}{v_{0}} \int d \mathbf{r}\left[W_{H}\left(\Phi_{H}-\hat{\phi}_{H}\right)+W_{T}\left(\Phi_{T}-\hat{\phi}_{T}\right)\right]\right\} .
\end{aligned}
$$

One can show that

$$
\exp \left[-\frac{1}{v_{0}} \int d \mathbf{r} W \hat{\phi}\right]=\prod_{i=1}^{n} \exp \left[\frac{v_{x}}{v_{0}} W\left(\mathbf{r}_{i}\right)\right]
$$

where $x=H$ or $T$. The partition function (A.8) is then

$$
\begin{aligned}
\mathcal{Z}= & \frac{\mathcal{N}}{n !} \int \mathcal{D} \Phi_{H} \mathcal{D} \Phi_{T} \mathcal{D} W_{H} \mathcal{D} W_{T} \prod_{i=1}^{n} d \mathbf{r}_{i}^{H} d \mathbf{r}_{i}^{T} \\
& \exp \left[-\frac{v_{H}}{v_{0}} W_{H}\left(\mathbf{r}_{i}^{H}\right)-\frac{v_{T}}{v_{0}} W_{T}\left(\mathbf{r}_{i}^{T}\right)-\mathcal{V}_{\text {conn }}\left(\mathbf{r}_{i}^{H}, \mathbf{r}_{i}^{T}\right)\right] \\
& \times \exp \left\{-\mathcal{V}\left[\Phi_{H}, \Phi_{T}\right]+\frac{1}{v_{0}} \int d \mathbf{r}\left(W_{H} \Phi_{H}+W_{T} \Phi_{T}\right)\right\}
\end{aligned}
$$

Let us define a single molecule partition function as

$$
Q \equiv \int d \mathbf{r} d \mathbf{r}^{\prime} G\left(\mathbf{r}, \mathbf{r}^{\prime}\right)
$$


where

$$
G\left(\mathbf{r}, \mathbf{r}^{\prime}\right)=\exp \left[-\frac{v_{H}}{v_{0}} W_{H}(\mathbf{r})-\frac{v_{T}}{v_{0}} W_{T}\left(\mathbf{r}^{\prime}\right)-\mathcal{V}_{\text {conn }}\left(\mathbf{r}, \mathbf{r}^{\prime}\right)\right]
$$

$\mathcal{Z}$ becomes

$$
\begin{aligned}
\mathcal{Z}= & \frac{\mathcal{N}}{n !} \int \mathcal{D} \Phi_{H} \mathcal{D} \Phi_{T} \mathcal{D} W_{H} \mathcal{D} W_{T} Q^{n} \\
& \times \exp \left\{-\mathcal{V}\left[\Phi_{H}, \Phi_{T}\right]+\frac{1}{v_{0}} \int d \mathbf{r}\left(W_{H} \Phi_{H}+W_{T} \Phi_{T}\right)\right\}
\end{aligned}
$$

where $Q$ has units of (volume) $)^{2}$. We make both $Q$ and $\mathcal{N}$ dimensionless using the reference volume $v_{0}$. Taking the mean field (saddle function) of this gives

$$
\mathcal{Z}=\frac{\mathcal{N}}{n !} Q^{n} \exp \left\{-\mathcal{V}\left[\Phi_{H}, \Phi_{T}\right]+\frac{1}{v_{0}} \int d \mathbf{r}\left(W_{H} \Phi_{H}+W_{T} \Phi_{T}\right)\right\} .
$$

The free energy is therefore

$$
\frac{F}{k_{B} T}=\ln n !-n \ln Q+\mathcal{V}\left[\varphi_{H}, \varphi_{T}\right]-\frac{1}{v_{0}} \int d \mathbf{r}\left(w_{H} \varphi_{H}+w_{T} \varphi_{T}\right)
$$

where constant terms are added and dropped as convenient. Dividing by $n$

$$
\frac{F}{n k_{B} T}=-\ln Q+\frac{\left(v_{H}+v_{T}\right)}{V} \mathcal{V}\left[\varphi_{H}, \varphi_{T}\right]-\frac{\left(v_{H}+v_{T}\right)}{V} \int \frac{d \mathbf{r}}{v_{0}}\left(w_{H} \varphi_{H}+w_{T} \varphi_{T}\right)
$$

since $n=V /\left(v_{H}+v_{T}\right)$ for a neat amphiphilic system where $V$ is the system volume.

Next, we need to choose the particle based potential $\mathcal{V}_{\text {conn }}\left(\mathbf{r}, \mathbf{r}^{\prime}\right)$ and the field based potential $\mathcal{V}\left[\varphi_{H}, \varphi_{T}\right]$. We start with the latter. It is comprised of two terms:

$$
\mathcal{V}\left[\varphi_{H}, \varphi_{T}\right]=\mathcal{V}_{H T}\left[\varphi_{H}, \varphi_{T}\right]+\mathcal{V}_{E O S}\left[\varphi_{H}, \varphi_{T}\right]
$$

The first is easily written in terms of the particle based potential of equation (A.1) and is

$$
\mathcal{V}_{H T}\left[\varphi_{H}, \varphi_{T}\right]=\int \frac{d \mathbf{r}}{v_{0}} \frac{d \mathbf{r}^{\prime}}{v_{0}} \varphi_{H}(\mathbf{r}) \mathcal{V}_{H T}\left(\left|\mathbf{r}-\mathbf{r}^{\prime}\right|\right) \varphi_{T}\left(\mathbf{r}^{\prime}\right)
$$

Substitution of equation(s) (A.2) into the instantaneous version (with carets) of equation (A.17) will return the original particle based potential term from (A.1). The mean field version looks the same but without the carets. One must choose the particle based potential between head and tail groups of different molecules. Relative to the head-head and tail-tail interactions, this should, from an incompressible point of view, be repulsive. We choose a Gaussian potential described by a prefactor $\mathcal{V}_{H T}^{0}$ and width $\mathcal{V}_{H T}^{w}$ which we found to behave well computationally. Specifically, it is

$$
\mathcal{V}_{H T}(r)=\mathcal{V}_{H T}^{0} \exp \left[\frac{-r^{2}}{\left(\mathcal{V}_{H T}^{w}\right)^{2}}\right] \text {. }
$$


The equation of state term is chosen as

$$
\mathcal{V}_{E O S}\left[\varphi_{H}, \varphi_{T}\right]=\frac{\kappa}{2} \int d \mathbf{r}\left[1-\varphi_{H}(\mathbf{r})-\varphi_{T}(\mathbf{r})\right]^{2}
$$

where $\kappa$ is a compressibility parameter that should be chosen as a large number to penalize, quadratically, any deviation from incompressibility. In block copolymer SCFT, an exact incompressibility is incorporated through an additional field, but this approach is numerically unstable for the present set of equations. The only remaining term is the connectivity term $\mathcal{V}_{\text {conn }}\left(\mathbf{r}, \mathbf{r}^{\prime}\right)$ that appears in the single molecule partition function $Q$ of equation (A.11). We use a simple spring potential

$$
\mathcal{V}_{\text {conn }}\left(\mathbf{r}, \mathbf{r}^{\prime}\right)=k\left(\left|\mathbf{r}-\mathbf{r}^{\prime}\right|-l\right)^{2}
$$

which, when placed in (A.11), gives another Gaussian. The spring constant $k$ should be chosen as stiff to suppress vibrational freedom but can be varied to model different amphiphilic molecules. $l$ is the equilibrium spacing between the centers of the head and tail groups. With these choices, the free energy (A.16) becomes

$$
\begin{aligned}
\frac{F}{n k_{B} T}= & -\ln Q+\frac{\left(v_{H}+v_{T}\right)}{V} \int \frac{d \mathbf{r}}{v_{0}}\left\{\int \frac{d \mathbf{r}^{\prime}}{v_{0}} \varphi_{H}(\mathbf{r}) \mathcal{V}_{H T}\left(\left|\mathbf{r}-\mathbf{r}^{\prime}\right|\right) \varphi_{T}\left(\mathbf{r}^{\prime}\right)\right. \\
& \left.+\frac{v_{0} \kappa}{2}\left[1-\varphi_{H}(\mathbf{r})-\varphi_{T}(\mathbf{r})\right]^{2}-w_{H}(\mathbf{r}) \varphi_{H}(\mathbf{r})-w_{T}(\mathbf{r}) \varphi_{T}(\mathbf{r})\right\} .
\end{aligned}
$$

We choose $v_{0} \equiv v_{H}+v_{T}$ as our reference volume, $\sigma \equiv v_{0}^{1 / 3}$ as our length scale (meaning that $l / \sigma$ is on the order of 1 ) and $\tilde{\kappa} \equiv v_{0} \kappa$ as a dimensionless compressibility parameter. The free energy then simplifies to

$$
\begin{aligned}
\frac{F}{n k_{B} T}= & -\ln Q+\frac{v_{0}}{V} \int \frac{d \mathbf{r}}{v_{0}}\left\{\int \frac{d \mathbf{r}^{\prime}}{v_{0}} \varphi_{H}(\mathbf{r}) \mathcal{V}_{H T}\left(\left|\mathbf{r}-\mathbf{r}^{\prime}\right|\right) \varphi_{T}\left(\mathbf{r}^{\prime}\right)\right. \\
& \left.+\frac{\tilde{\kappa}}{2}\left[1-\varphi_{H}(\mathbf{r})-\varphi_{T}(\mathbf{r})\right]^{2}-w_{H}(\mathbf{r}) \varphi_{H}(\mathbf{r})-w_{T}(\mathbf{r}) \varphi_{T}(\mathbf{r})\right\}
\end{aligned}
$$

The uniform limit of this is

$$
\frac{F}{n k_{B} T}=\phi_{H} \phi_{T} \int \frac{d \mathbf{r}}{v_{0}} \mathcal{V}_{H T}(\mathbf{r})-\ln \left[\frac{V}{v_{0}} \int \frac{d \mathbf{r}}{v_{0}} e^{-\mathcal{V}_{c o n n}(\mathbf{r})}\right]
$$

where $\phi_{H}$ and $\phi_{T}$ are the global volume fractions of heads and tails, respectively. To make our zero of free energy more similar to the block copolymer case, we can subtract off the 
total connectivity energy term. This has no effect on morphology because, operationally, this residual term doesn't appear in the SCFT equations. The free energy becomes

$$
\begin{aligned}
\frac{F}{n k_{B} T}= & -\ln Q+\frac{1}{V} \int d \mathbf{r}\left\{\int d \mathbf{r}^{\prime} \varphi_{H}(\mathbf{r}) \mathcal{V}_{H T}\left(\left|\mathbf{r}-\mathbf{r}^{\prime}\right|\right) \varphi_{T}\left(\mathbf{r}^{\prime}\right)\right. \\
& \left.+\frac{\tilde{\kappa}}{2}\left[1-\varphi_{H}(\mathbf{r})-\varphi_{T}(\mathbf{r})\right]^{2}-w_{H}(\mathbf{r}) \varphi_{H}(\mathbf{r})-w_{T}(\mathbf{r}) \varphi_{T}(\mathbf{r})\right\} \\
& +\ln \left[V \int d \mathbf{r} e^{-\mathcal{V}_{\text {conn }}(\mathbf{r})}\right]
\end{aligned}
$$

which is the same as equation (7) of the theory section. The uniform limit is now

$$
\frac{F}{n k_{B} T}=\phi_{H} \phi_{T} \int d \mathbf{r} \mathcal{V}_{H T}(\mathbf{r})
$$

which is the same as equation (8) of the theory section. In equations (A.24) and (A.25), we have simplified the presentation by absorbing the $v_{0}$ molecular volume into dimensionless distance units. Taking functional derivatives of (A.24) with respect to the volume fraction functions and chemical potential fields give the SCFT equations (1)-(6) of the theory section.

\section{Acknowledgments}

We acknowledge support from the University of Waterloo International Work Study Program. Some calculations were performed on the computing facilities of SHARCNET. RBT acknowledges helpful discussions with Prof. Bae-Yeun Ha.

[1] R. A. L. Jones, Soft Machines: Nanotechnology and Life (Oxford University Press, New York, 2004).

[2] P. Tanner, P. Baumann, R. Enea, O. Onaca, C. Palivan, and W. Meier, Accounts Chem. Res. 44, 1039 (2011).

[3] S. J. Holder and N. A. J. M. Sommerdijk, Polym. Chem. 2, 1018 (2011).

[4] I. W. Hamley, Nanotechnology 14, R39 (2003).

[5] M. J. Greenall and G. Gompper, Macromolecules 45, 525 (2012).

[6] L. Zhang, A. Sevink, and F. Schmid, Macromolecules 44, 9434 (2011).

[7] X. He and F. Schmid, Phys. Rev. Lett. 100, 137802 (2008).

[8] Y. Jiang, T. Chen, F. Ye, H. Liang, and A. C. Shi, Macromolecules 38, 6710 (2005). 
[9] Y. Yang, F. Qiu, P. Tang, and H. Zhang, Sci. China Ser. B 49, 21 (2006).

[10] R. G. Larson, Curr. Opin. Colloid In. 2, 361 (1997).

[11] F. Schmid and M. Schick, J. Chem. Phys. 102, 2080 (1995).

[12] F. Schmid, Phys. Rev. E 55, 5774 (1997).

[13] I. Szleifer, A. Ben-Shaul, and W. M. Gelbart, J. Chem. Phys 83, 3612 (1985).

[14] I. Szleifer, A. Ben-Shaul, and W. M. Gelbart, J. Chem. Phys 85, 5345 (1986).

[15] A. D. Mackie, A. Z. Panagiotopoulos, and I. Szleifer, Langmuir 13, 5022 (1997).

[16] C. B. E. Guerin and I. Szleifer, Langmuir 15, 7901 (1999).

[17] N. Zheng, J. Geehan, and M. D. Whitmore, Phys. Rev. E 75, 051922 (2007).

[18] M. D. Whitmore, J. P. Whitehead, and A. Roberge, Can. J. Phys. 76, 831 (1998).

[19] M. D. Whitmore and J. P. Whitehead, Can. J. Phys. 76, 883 (1998).

[20] J. M. H. M. Scheutjens and G. J. Fleer, J. Phys. Chem. 83, 1619 (1979).

[21] J. M. H. M. Scheutjens and G. J. Fleer, J. Phys. Chem. 84, 178 (1980).

[22] F. A. M. Leermakers, Y. S. Sdranis, J. Lyklema, and R. D. Groot, Colloid Surface A 85, 135 (1994).

[23] W. B. Lee, R. Mezzenga, and G. H. Fredrickson, Phys. Rev. Lett. 99, 187801 (2007).

[24] W. B. Lee, R. Mezzenga, and G. H. Fredrickson, J. Chem. Phys. 128, 074504 (2008).

[25] M. Müller and M. Schick, in Current Topics in Membranes 68: Membrane Fusion, edited by L. V. Chernomordik and M. M. Kozlov (Academic Press, Elsevier, 2011), pp. 295-323.

[26] M. Schick, J. Stat. Phys. 142, 1317 (2011).

[27] K. Katsov, M. Müller, and M. Schick, Biophys. J. 90, 915 (2006).

[28] K. Katsov, M. Müller, and M. Schick, Pramana-J. Phys. 64, 1127 (2005).

[29] K. Katsov, M. Müller, and M. Schick, Biophys. J. 87, 3277 (2004).

[30] J. Y. Lee and M. Schick, Biophys. J. 94, 1699 (2008).

[31] J. Y. Lee and M. Schick, J. Chem. Phys. 127, 075102 (2007).

[32] J. Y. Lee and M. Schick, Biophys. J. 92, 3938 (2007).

[33] K. C. Daoulas and M. Müller, Adv. Polym. Sci. 224, 197 (2010).

[34] V. V. Ginzburg, K. Chang, P. K. Jog, A. B. Argenton, and L. Rakesh, J. Phys. Chem. B 115, 4654 (2011).

[35] G. J. A. Sevink and A. V. Zvelindovsky, Macromolecules 38, 7502 (2005).

[36] J. G. E. M. Fraaije and G. J. A. Sevink, Macromolecules 36, 7891 (2003). 
[37] M. J. Greenall and G. Gompper, Langmuir 27, 3416 (2011).

[38] J. Katsarus, T. A. Harroun, J. Pencer, and M. P. Nieh, Naturwissenschaften 92, 355 (2005).

[39] M. W. Matsen and F. S. Bates, Macromolecules 29, 7641 (1996).

[40] R. B. Thompson, C. B. Park, and P. Chen, J. Chem. Phys 133, 144913 (2010).

[41] K. M. Hong and J. Noolandi, Macromolecules 14, 727 (1981).

[42] M. D. Whitmore and J. Noolandi, J. Chem. Phys 93, 2946 (1990).

[43] P. Stasiak and M. W. Matsen, Eur. Phys. J. E 34, 110 (2011).

[44] E. Helfand, J. Chem. Phys 62, 999 (2011).

[45] S. M. Hur, C. J. Garca-Cervera, and G. H. Fredrickson, Macromolecules 45, 2905 (2012).

[46] R. B. Thompson, K. Ø. Rasmussen, and T. Lookman, J. Chem. Phys 120, 31 (2004).

[47] R. B. Thompson, Phys. Rev. E 73, 020502(R) (2006).

[48] R. B. Thompson, Phys. Rev. E 74, 041501 (2006).

[49] K. Eriksson, C. Johnson, and A. Logg, SIAM J. Sci. Comput. 25, 1142 (2003).

[50] D. G. Anderson, J. Assoc. Comput. Mach. 12, 547 (1965).

[51] E. W. Cochran, C. J. Garcia-Cervera, and G. H. Fredrickson, Macromolecules 39, 2449 (2006).

[52] G. C. Shearman, B. J. Khoo, M. L. Motherwell, K. A. Brakke, O. Ces, C. E. Conn, J. M. Seddon, and R. H. Templer, Langmuir 23, 7276 (2007).

[53] M. W. Matsen, J. Chem. Phys. 104, 7758 (1996).

[54] M. W. Matsen and C. Barrett, J. Chem. Phys. 109, 4108 (1998).

[55] R. Hołyst and P. Oswald, Macromol. Theory Simul. 10, 1 (2001).

[56] D. Düchs and D. E. Sullivan, J. Phys.: Condens. Matter 14, 12189 (2002).

[57] A. J. Spakowitz and Z.-G. Wang, J. Chem. Phys. 119, 13113 (2003).

[58] V. Pryamitsyn and V. Ganesan, J. Chem. Phys. 120, 5824 (2004).

[59] R. C. Hidalgo, D. E. Sullivan, and J. Z. Y. Chen, Phys. Rev. E 71, 041804 (2005).

[60] B. D. Olsen, M. Shah, V. Ganesan, and R. A. Segalman, Macromolecules 41, 6809 (2008).

[61] W. Song, P. Tang, H. Zhang, Y. Yang, and A.-C. Shi, Macromolecules 42, 6300 (2009).

[62] Y. Jiang and J. Z. Y. Chen, Macromolecules 43, 10668 (2010).

[63] J. Gao, W. Song, P. Tang, and Y. Yang, Soft Matter 7, 5208 (2011).

[64] C. A. Tyler and D. C. Morse, Phys. Rev. Lett. 94, 208302 (2005).

[65] There were some runs that did not converge using our chosen settings for Anderson iteration. These cases were examined individually by adjusting the Anderson settings where they 
were needed to clarify the phase behaviour. If other runs provided enough information, the unconverged runs were merely abandoned as superfluous.

[66] There were rare cases where it was difficult to distinguish whether a morphology was G or PL. Both are threefold connected structures and are very similar in free energy. Defects in large boxes or box size limitations in small boxes could, in any case, affect the final determination. Tyler and Morse have observed another threefold connected structure, the $O^{70}$ phase, which could also be present [64]. Given the qualitative nature of the present study, it is not important which complex three point coordinated structure is marginally more stable than the others. 